\title{
Adjuvant combined systemic chemotherapy and intraperitoneal chemotherapy for locally advanced gastric cancer
}

\author{
SHENG-LIU XUE ${ }^{1}$, HUA-FANG SU ${ }^{1}$, XIAO-QU HU ${ }^{2}$, XIA DENG $^{1}$, MEI-LONG HU ${ }^{1}$ and CONG-YING XIE ${ }^{1}$ \\ Departments of ${ }^{1}$ Radiation Oncology and Chemotherapy and ${ }^{2}$ Surgery, \\ The First Affiliated Hospital of Wenzhou Medical College, Wenzhou, Zhejiang 325002, P.R. China
}

Received May 24, 2012; Accepted September 6, 2012

DOI: $10.3892 / \mathrm{ol} .2012 .914$

\begin{abstract}
The optimal adjuvant treatment modality for gastric cancer has not been well defined. The aim of this study was to evaluate the efficacy and feasibility of adjuvant combined systemic and intraperitoneal chemotherapy (ACSIP) in high-risk patients with locally advanced gastric cancer. Between June 2003 and December 2008, 62 eligible patients with serosa-infiltrating and/or node-positive gastric cancer following curative gastrectomy with D2 lymphadenectomy received ACSIP, consisting of intravenous oxaliplatin $85 \mathrm{mg} / \mathrm{m}^{2}$ on day 1 followed by leucovorin (LV) $200 \mathrm{mg} / \mathrm{m}^{2}$ and 5-fluorouracil (5-FU) $450 \mathrm{mg} / \mathrm{m}^{2}$ on days $1-3$, intraperitoneal 5-FU $600 \mathrm{mg} / \mathrm{m}^{2}$ on days 4-5 and cisplatin (CDDP) $40 \mathrm{mg} / \mathrm{m}^{2}$ on day 5 . Survival rates, the sites of first treatment failure and safety were analyzed. At a median follow-up of 45 months (range 7-101), the 3-year disease-free survival (DFS) and overall survival (OS) rates were 66.1 and $74.2 \%$, respectively. Initial peritoneal and hepatic failures were found in $6(24.0 \%)$ and $3(12.0 \%)$ of the 25 patients with recurrence, respectively. Neutropenia, gastrointestinal side effects and peripheral neuropathy were the most common grade 3-4 toxicities; however, they were all infrequent and manageable. No serious surgical complications or treatment-related mortality was observed. The results of this study indicate that ACSIP is effective and feasible for locally advanced gastric cancer with encouraging survival rates and possibly decreased peritoneal and hepatic recurrences. The benefits of this promising combined adjuvant treatment modality warrant further studies.
\end{abstract}

\footnotetext{
Correspondence to: Dr Cong-Ying Xie, Department of Radiation Oncology and Chemotherapy, The First Affiliated Hospital of Wenzhou Medical College, 2 Fuxuexiang Road, Wenzhou, Zhejiang 325002, P.R. China

E-mail: congyingxie@163.com
}

Key words: locally advanced gastric cancer, adjuvant chemotherapy, combined therapy, intraperitoneal chemotherapy

\section{Introduction}

Gastric cancer is the second most common cancer and the third leading cause of mortality from cancer in China (1). There are more new cases diagnosed in China than in any other country each year. Complete surgical resection is the only potential curative treatment for gastric cancer. Nonetheless, the longterm results of resectable gastric cancer are still poor. More than $50 \%$ of patients will develop locoregional recurrences and/or distant metastases within 1-3 years of curative surgery and patients with serosa-infiltrating and/or node-positive cancer are at a particularly high risk of recurrence $(2,3)$. Levels of interest in adjuvant treatment have increased in the past few decades. In spite of the notable improvement demonstrated by Intergroup 116 (adjuvant chemoradiation) (4), MAGIC (neoadjuvant and adjuvant chemotherapy) (5) and ACTS-GC (adjuvant S1) trials (6), almost half of gastric cancer patients will succumb to the condition within three years of surgery. These trials all showed the same pattern of cancer recurrence. The patients were treated either by surgery alone or surgery plus peri- or postoperative chemoradiotherapy; however, peritoneal failure remained the most common pattern of first recurrence (7). Therefore, it is essential to reinforce efforts to find more effective adjuvant treatment modalities.

Systemic chemotherapy (SCT) possesses the theoretical advantage of treating undetectable cancer cells that have spread systemically at the time of surgery and will ultimately represent a major source of treatment failure for patients with resectable gastric cancer. Recent meta-analyses (8-10) have demonstrated that adjuvant SCT is effective in treating gastric cancer. In addition, intraperitoneal chemotherapy (IPCT) has several unique pharmacokinetic advantages which are able to eradicate free cancer cells and micrometastatic or microscopic residual focus in the peritoneal cavity. IPCT also reduces recurrence and therefore combines the effects of intraportal chemotherapy on the liver with a direct effect on the peritoneum and the resection site. Adjuvant IPCT has also been shown to be a valuable strategy in treating gastric cancer (11-13). Therefore, cumulative data suggests that benefits may be obtained by combining SCT and IPCT to decrease both local and distant metastases simultaneously with different targets, different times of effect and non-overlapping side effects. Taken together, SCT and IPCT appear well-suited for combined use; therefore, it is rational to combine SCT with 
IPCT in an adjuvant setting for gastric cancer (13). Moreover, the benefit of this combined strategy has been verified by many convincing clinical trials in ovarian cancer (14).

Therefore, we conducted a study to evaluate the efficacy and feasibility of this novel adjuvant chemotherapy approach in high-risk patients with locally advanced gastric cancer. This study was approved by the ethics committees of The First Affiliated Hospital of Wenzhou Medical College.

\section{Materials and methods}

Patients. All patients enrolled were required to have adenocarcinoma of the stomach confirmed histologically and undergo a curative resection with D2 lymphadenectomy and negative margins (R0 resection). Enrolled patients were also required to have serosa-infiltrating (pT3+ stage) and/or regional lymph node-positive $(\mathrm{pN}+)$ cancer with no distant metastases (stage II through IVM0, according to the 2002 staging criteria of the American Joint Commission on Cancer) (15). Eligibility was determined by reviewing the study forms, operative and pathology reports carefully. Patients with any overt evidence of peritoneal spread were excluded. Additional requirements for eligibility were an Eastern Cooperative Oncology Group performance status $\leq 1$; age of 20 -80 years; adequate hematological levels (neutrophils $\geq 2 \times 10^{9} / 1$; hemoglobin $\geq 900 \mathrm{~g} / \mathrm{l}$; platelets $\geq 100 \times 10^{9} / 1$ ); hepatic function (bilirubin $\leq 25 \mu \mathrm{mol} / 1$; aspartate aminotransferase and alanine aminotransferase $\leq 2.5 \times$ upper normal limit); renal function (creatinine $\leq 130 \mu \mathrm{mol} / \mathrm{l}$ ); cardiac function (New York Heart Association class <II); no previous malignancies other than superficial skin cancer or in situ cervical carcinoma and no previous treatments such as neoadjuvant chemotherapy or radiotherapy. Patients were enrolled in this study no earlier than 1 week and no later than 3 weeks after surgery. Metastatic disease was ruled out by computed tomography (CT) scans of the brain, chest and whole abdomen prior to surgery. All patients signed an informed consent prior to treatment.

Treatment. ACSIP started within 1-3 weeks of surgery. The course of treatment was repeated every 28 days for up to 6 cycles. SCT consisted of intravenous oxaliplatin $85 \mathrm{mg} / \mathrm{m}^{2}$ on day 1 over a 2-h period followed by LV $200 \mathrm{mg} / \mathrm{m}^{2}$ and 5 -FU $450 \mathrm{mg} / \mathrm{m}^{2}$ for 3 days (days 1 to 3 ). Prior to every IPCT, peritoneal catheterization was performed blindly under local anesthesia using the Seldinger technique based on previous ultrasonography localization of the puncture site. Intraperitoneal drug delivery was administered through a single-lumen central venous catheter (Arrow International, Inc., Reading, PA, USA) inserted into the abdominal cavity. IPCT consisted of 5 -FU $600 \mathrm{mg} / \mathrm{m}^{2}$ on days 4 to 5 followed by CDDP $40 \mathrm{mg} / \mathrm{m}^{2}$ on day 5. Prior to IPCT, $100 \mathrm{ml} 0.9 \%$ normal saline with $10 \mathrm{mg}$ dexamethasone was instilled into the peritoneal cavity. 5-FU and CDDP were diluted in 1,000 $\mathrm{ml}$ and $500 \mathrm{ml} \mathrm{0.9 \%} \mathrm{normal} \mathrm{saline,} \mathrm{respectively.} \mathrm{Patients} \mathrm{were} \mathrm{asked}$ to change their body position every $15 \mathrm{~min}$ for $2 \mathrm{~h}$ following the intraperitoneal administration. The fluid was left in the peritoneal cavity until the next infusion. The catheter was removed $24 \mathrm{~h}$ after the last infusion. If grade 3-4 hematological toxicity occurred, the doses of SCT were decreased by $20 \%$. If grade 3-4 non-hematological toxicity occurred, or if toxicity
Table I. Patient and tumor characteristics $(n=62)$.

Characteristics No. of patients $(\%)$

\begin{tabular}{lc}
\hline Gender & \\
Male & $45(72.6)$ \\
Female & $17(27.4)$ \\
Age (years) & 56 \\
Median & $32-77$ \\
Range & \\
Tumor location & $12(19.4)$ \\
Upper third of stomach & $39(62.9)$ \\
Middle third of stomach & $11(17.7)$ \\
Lower third of stomach & \\
Type of gastrectomy & $42(67.7)$ \\
Subtotal & $20(32.3)$ \\
Total & \\
Histological type & $2(3.2)$ \\
Well-differentiated & $16(25.8)$ \\
Moderately differentiated & $44(71.0)$ \\
Poorly differentiated & \\
Pathological T stage & $1(1.6)$ \\
pT1 & $9(14.5)$ \\
pT2 & $51(82.3)$ \\
pT3 & $1(1.6)$ \\
pT4 &
\end{tabular}

Pathological node status

NO

$\mathrm{N} 1$

$41(66.1)$

$\mathrm{N} 2$

$9(14.5)$

AJCC stage (2002)

II

$19(30.6)$

IIIA

$30(48.4)$

IIIB

IV

(hematological or non-hematological) persisted for more than 2 weeks, the doses of SCT and IPCT were both decreased by $20 \%$.

Follow-up evaluation. Comprehensive baseline assessments, including a complete medical history, physical examination, complete blood cell count, blood chemistries, tumor markers (CEA, CA19.9 and CA125), and brain, chest and whole abdomen CT scans were conducted prior to both surgery and treatment for every patient. During the treatment, patients were checked from weekly to monthly intervals by physical examination, toxicity assessment and blood examination. Toxicities were evaluated following the National Cancer Institute Common Toxicity Criteria, version 2.0. Follow-up was performed at 3-month intervals for 2 years, 6-month intervals for 3 years and yearly thereafter. Follow-up examinations consisted of physical examination, complete blood 
Table II. Sites of first treatment failure $(n=25)$.

\begin{tabular}{lc}
\hline Sites & No. of patients $(\%)$ \\
\hline Locoregional & $5(20.0)$ \\
Peritoneal & $6(24.0)$ \\
Hepatic & $3(12.0)$ \\
Other distant metastases & $11(44.0)$
\end{tabular}

cell count, blood chemistries, tumor markers and abdominopelvic ultrasonography and/or CT. Chest X-rays or CT scans, if indicated clinically, were performed every 6 months in the first 3 years then yearly to ensure recurrence data collection. A radionuclide bone scan and endoscopy were performed if clinically indicated. The sites and date of first relapse and the date of death, if the patient succumbed to their condition, were recorded. Recurrence was confirmed by clinical, radiological and histological examinations (whenever feasible). Typical nodules in liver or lung with imaging studies or typical lesions in the radionuclide bone scan and MRI or plain X-ray were accepted as relapse without histological confirmation. Patterns of recurrence reported in this study represent first sites of documented recurring disease following curative resection, including locoregional, peritoneal, hepatic and other distant metastasis. Disease-free survival (DFS) and overall survival (OS) rates were measured from the date of surgery. DFS was measured to the date of recurrence and OS was defined as the time from surgery to the date of death from any cause. DFS and OS rates were estimated using the Kaplan-Meier method.

\section{Results}

Patient characteristics. Between June 2003 and December 2008, a total of 67 patients were enrolled in the study. Five patients $(7.5 \%)$ were excluded from the analysis: one had a positive surgical margin; one refused to start treatment; two were lost to follow-up evaluation during ACSIP and one had protocol violation, thus the analyses were carried out in the remaining 62 eligible patients. For the 62 patients, the median age was 56 years (range 32-77) with 45 males and 17 females. The majority of patients had serosa-infiltrating $(83.9 \%)$ and/or node-positive $(87.1 \%)$ cancer (Table I).

Survival. At the time of survival analysis, the median follow-up time for this study was 45 months (range 7-101). At present, 37 patients are still alive, with 35 of them free of recurrence. There are 2 patients with documented recurrence who are still alive. During follow-up, 25 patients succumbed to their condition and 2 of them succumbed without evidence of recurrence or metastasis (one from cardiovascular disease and the other for unknown reasons). The 3-year DFS rate was $66.1 \%$ (Fig. 1). The 3-year OS rate was $74.2 \%$ (Fig. 2).

Relapse. During follow-up, 25 patients (40.3\%) eventually relapsed and 23 patients succumbed to disease recurrence. As it concerns the sites of first treatment failure, among the 25 patients, $6(24.0 \%)$ and $3(12.0 \%)$ of them experienced

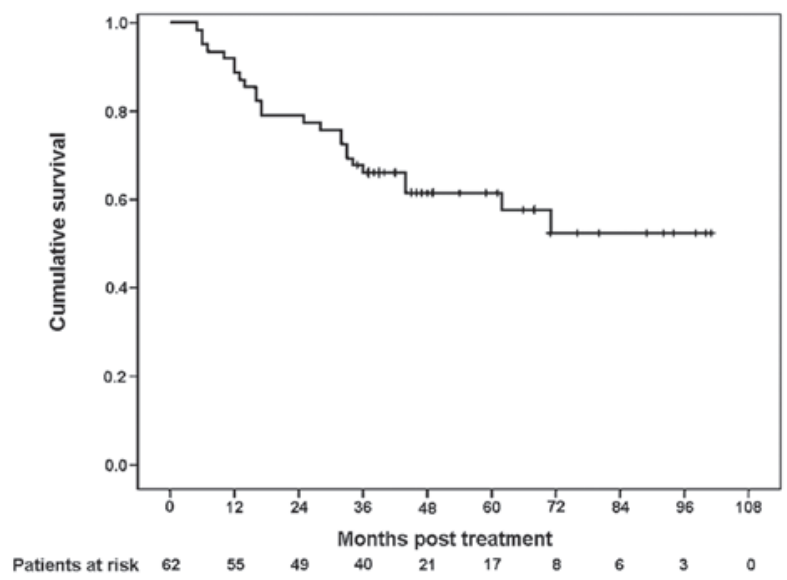

Figure 1. Kaplan-Meier survival curve for disease-free survival (DFS). The 3-year DFS rate was $66.1 \%$.

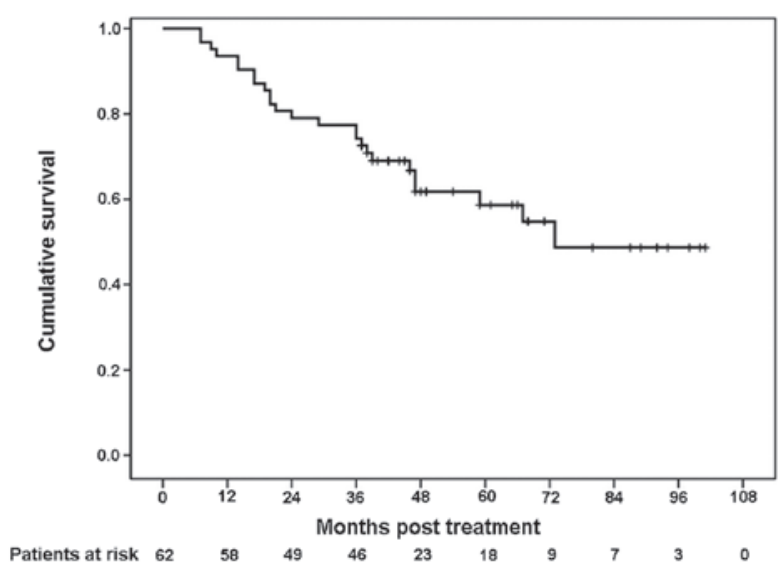

Figure 2. Kaplan-Meier survival curve for overall survival (OS). The 3-year OS rate was $74.2 \%$

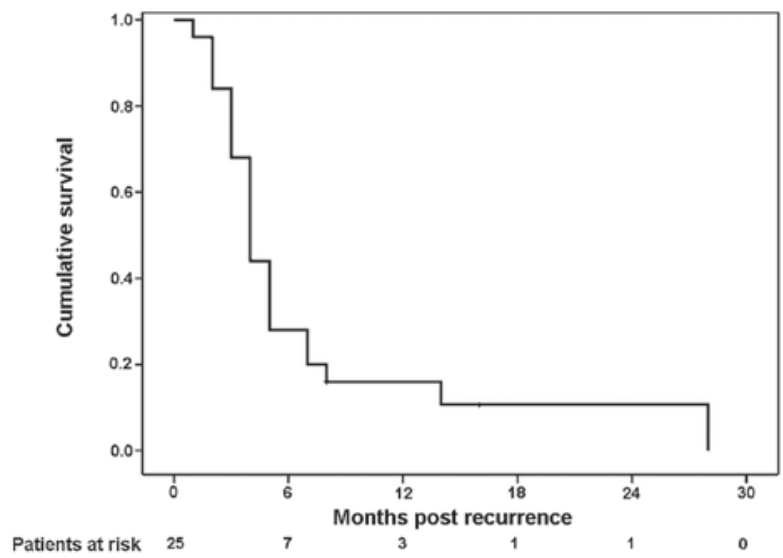

Figure 3. Kaplan-Meier survival curve for survival following recurrence. The median time from recurrence to death was 4 months, and $91.3 \%$ of patients succumbed to their condition within 1 year of the diagnosis of recurrence.

initial peritoneal and hepatic recurrence, respectively, 5 patients $(20.0 \%)$ had locoregional relapse and 11 patients $(44.0 \%)$ had other distant metastases (Table II). Twenty-one patients $(91.3 \%)$ succumbed to their condition within 1 year of 
Table III. Toxicities from treatment $(n=62)$.

\begin{tabular}{|c|c|c|c|}
\hline Toxicities & Grade 1-2, n (\%) & Grade 3-4, n (\%) & All,n (\%) \\
\hline \multicolumn{4}{|l|}{ Hematological } \\
\hline Neutropenia & $9(14.5)$ & $5(8.1)$ & $14(22.6)$ \\
\hline Anemia & $11(17.7)$ & 0 & $11(17.7)$ \\
\hline Thrombocytopenia & $10(16.1)$ & 0 & $10(16.1)$ \\
\hline \multicolumn{4}{|l|}{ Non-hematological } \\
\hline Stomatitis & $4(6.5)$ & 0 & $4(6.5)$ \\
\hline Diarrhea & $5(8.1)$ & $3(4.8)$ & $8(12.9)$ \\
\hline Nausea & $4(6.5)$ & $1(1.6)$ & $5(8.1)$ \\
\hline Vomiting & $5(8.1)$ & $1(1.6)$ & $6(9.7)$ \\
\hline Distention & $3(4.8)$ & 0 & $3(4.8)$ \\
\hline Abdominal pain & $1(1.6)$ & 0 & $1(1.6)$ \\
\hline Impaired liver function & $3(4.8)$ & 0 & $3(4.8)$ \\
\hline Impaired renal function & $2(3.2)$ & 0 & $2(3.2)$ \\
\hline Peripheral neuropathy & $6(9.7)$ & $1(1.6)$ & $7(11.3)$ \\
\hline
\end{tabular}

the diagnosis of recurrence. The median time from recurrence to death was 4 months (Fig. 3).

Toxicity and surgical complications. Overall, adverse toxicities were relatively uncommon and they were generally mild to moderate. Neutropenia $(8.1 \%)$, diarrhea $(4.8 \%)$, nausea (1.6\%), vomiting (1.6\%) and peripheral neuropathy (1.6\%) were the most common grade 3-4 toxicities. No treatment-related mortality was observed (Table III). Surgical complications were observed in 5 out of 62 patients $(8.1 \%)$ : anastomotic leakage in 2; intra-abdominal abscess in 1; wound infection in 1 and pancreatitis in 1 . Treatment was delayed in 6 of the 62 patients $(9.7 \%)$ due to toxicities and/or surgical complications; however, the toxicities and surgical complications were all manageable. Fifty-five $(88.7 \%)$ out of the 62 patients completed more than 4 cycles of ACSIP and 48 patients $(77.4 \%)$ completed 6 cycles. In addition, 4 patients completed 3 cycles of ACSIP, and 3 patients completed 2 cycles. The primary reasons for 14 patients (22.6\%) not completing 6 cycles of ACSIP were patient refusal (6 patients), disease progression (2 patients), toxicities (1 patient), surgical complications (1 patient), catheter-related complications (1 patient) and other reasons (3 patients).

\section{Discussion}

In the past decades, studies on adjuvant SCT for the treatment of resectable gastric cancer have shown conflicting results (8). Adjuvant SCT has not become the standard of care for gastric cancer except with S1 in Japan following the publication of the results of the ACTS-GC trial $(6,16)$. In addition, there is limited good quality evidence to determine the role of adjuvant IPCT in gastric cancer (17). The optimal adjuvant chemotherapy approach for gastric cancer such as SCT, IPCT alone or in combination has not yet been well-defined and there is no widely accepted chemotherapeutic regimen. Based on the recurrence patterns following curative resection, the rationale and advantage for combined SCT and IPCT in an adjuvant setting for gastric cancer is evident. In addition, the efficacy and feasibility of combined SCT and IPCT have been well-established in other intra-abdominal malignancies including colon (18) and ovarian cancer (14), particularly in the latter, for which convincing clinical trials have confirmed the improvement in survival.

Despite the attractive rationale for the combination of adjuvant SCT and IPCT, clinical experience with this treatment modality in gastric cancer is limited. To our knowledge, there were only a few earlier studies available exploring the use of ACSIP in gastric cancer. The earliest was by Atiq et al (19), published in 1993. This group assessed postoperative adjuvant intraperitoneal CDDP and 5-FU and systemic 5-FU chemotherapy in patients with resected gastric cancer. In that study, 16 out of 35 patients experienced recurrence, and 18 remained alive free of disease with a 3-year survival rate of $50.0 \%$. The treatments were also associated with altered metastatic patterns (decreased incidence of peritoneal spread and liver metastases). In another study by Zuo et al (20), a statistically significant improvement in 3-year survival was reported for the adjuvant intraperitoneal hyperthermic chemoperfusion combined with intravenous chemotherapy group; the 3-year survival rate was $83.0 \%$ in the combined group compared with $61.0 \%$ in the adjuvant intravenous chemotherapy alone group $(\mathrm{P}<0.05)$. Recently, a retrospective study by Shi et al (21) revealed that the 5-year OS rate in the adjuvant systemic chemotherapy combined with intraperitoneal perfusion chemotherapy (IP+) group was significantly better than that in the systemic chemotherapy only (IP-) group (60.4 vs. $42.9 \%$; $\mathrm{P}=0.001)$. In addition, the average progression-free survival in the IP+ group was significantly longer than that in the IP- group (60.5 vs. 46.2 months; $\mathrm{P}=0.001)$. Although the different patient selection criteria and chemotherapy regimens tested make the comparison between these studies and the present study difficult, the results of these studies and ours are both encouraging, as the 3 -year DFS rate was $66.1 \%$ and the 3 -year OS rate was $74.2 \%$ in the present study. However, the benefit of this promising 
combined adjuvant treatment modality should be confirmed in prospective randomized controlled trials.

The incidences of hepatic and peritoneal failure were relatively low in this study. An initial intraperitoneal and hepatic relapse was noted in $6(24.0 \%)$ and $3(12.0 \%)$ out of 25 patients with recurrence in this study, respectively, thus the incidences are lower than those revealed in the study of $\mathrm{Wu}$ et al (2), which addressed recurrence patterns following intended curative surgery in 611 patients with gastric cancer. In that study, 38.2 and $17.9 \%$ patients experienced peritoneal and hepatic recurrence, respectively. The low incidence of hepatic and peritoneal failure in this study is possibly related to the additive effects of systemic, intravenous and intraperitoneal drug administration. However, in the present study, $91.3 \%$ of patients succumbed to their condition within 1 year of the diagnosis of recurrence being made, and the median time from recurrence to mortality was only 4 months. These results are poorer than those of D'Angelica et al (22). They reported that $70.0 \%$ of patients succumbed to their condition within 1 year of the diagnosis of recurrence and the median time from recurrence to mortality was 6 months. In multivariate analysis, they demonstrated that shortened median time until mortality was significantly correlated with a higher T stage (4 vs. 7 months, $\mathrm{P}=0.007$ ) and involved lymph nodes (5 vs. 9 months, $\mathrm{P}=0.01$ ). In the present study, $83.9 \%$ of patients had serosa-infiltrating cancer and $87.1 \%$ of them were lymph node-positive, which may be the main causes of the short time to death following recurrence.

Patient tolerance of ACSIP was excellent in this study. On the whole, toxicities were mild to moderate. Neutropenia, gastrointestinal side effects and peripheral neuropathy were the most common grade 3-4 toxicities; however, they were all infrequent and manageable. There were no treatmentrelated mortalities. Chemical peritonitis had been reported in patients with intra-abdominal cancer receiving intraperitoneal high doses of 5-FU and CDDP for long periods (23); however, this did not occur in the present study as 5-FU and CDDP were administered for a shorter period of time. Several studies have suggested that there was an increase in morbidity and mortality with adjuvant perioperative intraperitoneal chemotherapy in gastric cancer. Yu et al (24) reported that the overall morbidity and mortality rates in patients receiving early postoperative IPCT starting on postoperative day 1 (the study group) were both higher than in those who underwent surgery only (the control group), although the difference was not significant ( 28 vs. $20.3 \%, \mathrm{P}=0.121 ; 5.6$ vs. $0.8 \%, \mathrm{P}=0.299$, respectively). By contrast, intra-abdominal sepsis without anastomotic leakage $(\mathrm{P}=0.008)$ and bleeding $(\mathrm{P}=0.002)$ occurred significantly more often in the study group compared with the control group. In a study by Rosen et al (25), significantly higher postoperative complication and mortality rates were also observed in patients receiving intraperitoneal mitomycin bound to activated carbon particles compared with the surgical control group (35 vs. $16.0 \%$; 11 vs. $2.0 \%$, respectively), thus the protocol committee decided to stop further recruitment of patients into that particular study. However, the incidence of surgical complications was only $8.1 \%$ in the present study, which was much lower than that in the above-mentioned studies. The difference in incidence between these studies and ours may be related to the different cancer stage, type of surgery, timing of IPCT commencement and the chemotherapeutic agents intraperitoneally administered. This study indicates that adjuvant intraperitoneal 5-FU and CDDP delivered at 1-3 weeks postoperatively may not increase the rates of surgical complications and mortality in patients with gastric cancer. In addition, 55 patients $(88.7 \%)$ completed more than 4 cycles of ACSIP and 48 patients (77.4\%) completed 6 cycles in the present study. It is possible that the benefit of ACSIP may be greater if more patients successfully complete 6 cycles of treatment; however, the effect of the duration of treatment on clinical outcome has not been confirmed by any study.

A considerable amount of research into adjuvant chemotherapy in gastric cancer is still necessary. Further improvements will require the development of more effective treatment modalities and chemotherapeutic regimens. During the last decade, several new agents including paclitaxel, docetaxel, oxaliplatin, irinotecan, capecitabine and S1 have shown promising activity in gastric cancer. From a theoretical point of view, regimens with higher activity may have more efficacy as adjuvant therapies $(26,27)$. However, the selection, dosage, combination, schedule of chemotherapy agents and the route and timing of administration need extensive testing in confirmatory studies.

In conclusion, the results of this study indicate that ACSIP is effective and feasible in high risk patients with locally advanced gastric cancer following curative gastrectomy under D2 lymphadenectomy and has encouraging 3-year DFS and OS rates. Failure pattern data suggest possible decreased peritoneal and hepatic recurrences. The benefit of this promising combined adjuvant treatment modality for this challenging disease warrants further studies.

\section{Acknowledgements}

The authors acknowledge Dr Jing Zhao for her assistance in the statistical analysis of the data, as well as Mr. Xian-Ce Jin for his revision of the manuscript.

\section{References}

1. Zhao P, Dai M, Chen W and Li N: Cancer trends in China. Jpn J Clin Oncol 40: 281-285, 2010.

2. Wu CW, Lo SS, Shen KH, et al: Incidence and factors associated with recurrence patterns after intended curative surgery for gastric cancer. World J Surg 27: 153-158, 2003.

3. Sugarbaker PH, Yu W and Yonemura Y: Gastrectomy, peritonectomy, and perioperative intraperitoneal chemotherapy: the evolution of treatment strategies for advanced gastric cancer. Semin Surg Oncol 21: 233-248, 2003.

4. Macdonald JS, Smalley SR, Benedetti J, et al: Chemoradiotherapy after surgery compared with surgery alone for adenocarcinoma of the stomach or gastroesophageal junction. N Engl J Med 345: 725-730, 2001

5. Cunningham D, Allum WH, Stenning SP, et al: Perioperative chemotherapy versus surgery alone for resectable gastroesophageal cancer. N Engl J Med 355: 11-20, 2006.

6. Sasako M, Sakuramoto S, Katai H, et al: Five-year outcomes of a randomized phase III trial comparing adjuvant chemotherapy with S-1 versus surgery alone in stage II or III gastric cancer. J Clin Oncol 29: 4387-4393, 2011.

7. Yang XJ, Huang CQ, Suo T, et al: Cytoreductive surgery and hyperthermic intraperitoneal chemotherapy improves survival of patients with peritoneal carcinomatosis from gastric cancer: final results of a phase III randomized clinical trial. Ann Surg Oncol 18: 1575-1581, 2011. 
8. GASTRIC (Global Advanced/Adjuvant Stomach Tumor Research International Collaboration) Group, Paoletti X, Oba K, et al: Benefit of adjuvant chemotherapy for resectable gastric cancer: a meta-analysis. JAMA 303: 1729-1737, 2010.

9. Sun P, Xiang JB and Chen ZY: Meta-analysis of adjuvant chemotherapy after radical surgery for advanced gastric cancer. Br J Surg 96: 26-33, 2009.

10. Liu TS, Wang Y, Chen SY and Sun YH: An updated metaanalysis of adjuvant chemotherapy after curative resection for gastric cancer. Eur J Surg Oncol 34: 1208-1216, 2008.

11. Xu DZ,Zhan YQ, Sun XW, Cao SM and Geng QR: Meta-analysis of intraperitoneal chemotherapy for gastric cancer. World J Gastroenterol 10: 2727-2730, 2004.

12. Yan TD, Black D, Sugarbaker PH, Zhu J, Yonemura Y, Petrou G and Morris DL: A systematic review and meta-analysis of the randomized controlled trials on adjuvant intraperitoneal chemotherapy for resectable gastric cancer. Ann Surg Oncol 14: 2702-2713, 2007.

13. Sugarbaker PH: Adjuvant intraperitoneal chemotherapy for advanced primary gastric cancer. Scand J Surg 95: 270-273, 2006.

14. Armstrong DK, Bundy B, Wenzel L, et al: Intraperitoneal cisplatin and paclitaxel in ovarian cancer. N Engl J Med 354: 34-43, 2006.

15. American Joint Committe on Cancer (AJCC): Cancer staging handbook: TNM classification of malignant tumours. 6th edition. Springer, New York, pp99-106, 2002.

16. Sano T and Aiko T: New Japanese classifications and treatment guidelines for gastric cancer: revision concepts and major revised points. Gastric Cancer 14: 97-100, 2011.

17. Matharu G, Tucker O and Alderson D: Systematic review of intraperitoneal chemotherapy for gastric cancer. Br J Surg 98: 1225-1235, 2011

18. Scheithauer W, Kornek GV, Marczell A, et al: Combined intravenous and intraperitoneal chemotherapy with fluorouracil + leucovorin vs fluorouracil + levamisole for adjuvant therapy of resected colon carcinoma. Br J Cancer 77: 1349-1354, 1998.
19. Atiq OT, Kelsen DP, Shiu MH, et al: Phase II trial of postoperative adjuvant intraperitoneal cisplatin and fluorouracil and systemic fluorouracil chemotherapy in patients with resected gastric cancer. J Clin Oncol 11: 425-433, 1993.

20. Zuo Y, Xu M, Shen D, Lu WD and Lu JF: Postoperative intraperitioneal hyperthermic chemoperfusion combined with intravenous chemotherapy for 82 advanced gastric cancer patients. Zhonghua Zhong Liu Za Zhi 26: 247-249, 2004.

21. Shi C, Yang B, Chen Q, Yang J and Fan N: Retrospective analysis of adjuvant intraperitoneal chemotherapy effect prognosis of resectable gastric cancer. Oncology 80: 289-295, 2011.

22. D'Angelica M, Gonen M, Brennan MF, Turnbull AD, Bains M and Karpeh MS: Patterns of initial recurrence in completely resected gastric adenocarcinoma. Ann Surg 240: 808-816, 2004.

23. Schilsky RL, Choi KE, Grayhack J, Grimmer D, Guarnieri C and Fullem L: Phase I clinical and pharmacologic study of intraperitoneal cisplatin and fluorouracil in patients with advanced intraabdominal cancer. J Clin Oncol 8: 2054-2061, 1990.

24. Yu W, Whang I, Averbach A, Chang D and Sugarbaker PH: Morbidity and mortality of early postoperative intraperitoneal chemotherapy as adjuvant therapy for gastric cancer. Am Surg 64: 1104-1108, 1998.

25. Rosen HR, Jatzko G, Repse S, et al: Adjuvant intraperitoneal chemotherapy with carbon-adsorbed mitomycin in patients with gastric cancer: results of a randomized multicenter trial of the Austrian Working Group for Surgical Oncology. J Clin Oncol 16: 2733-2738, 1998.

26. De Vita F, Vecchione L, Galizia G, et al: Perspectives in adjuvant therapy of gastric cancer. Oncology 77: 38-42, 2009.

27. Bittoni A, Maccaroni E, Scartozzi M, Berardi R and Cascinu S: Chemotherapy for locally advanced and metastatic gastric cancer: state of the art and future perspectives. Eur Rev Med Pharmacol Sci 14: 309-314, 2010. 